\title{
Controlling activated surface diffusion by external fields
}

\author{
Peter Talkner ${ }^{\text {a }}$, Eli Hershkovitz ${ }^{\mathrm{b}}$, Eli Pollak ${ }^{\mathrm{b}, *}$, Peter Hänggi ${ }^{\mathrm{c}}$ \\ a Paul Scherrer Institute, CH 5232 Villigen, Switzerland \\ ${ }^{\mathrm{b}}$ Chemical Physics Department, Weizmann Institute of Science, 76100 Rehovot, Israel

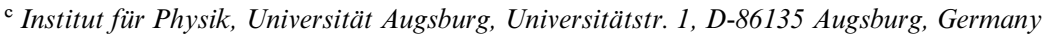

Received 23 March 1999; accepted for publication 27 May 1999

\begin{abstract}
A theory is presented for the diffusion coefficient and the hopping distribution of an adatom on a surface in the presence of external fields. Relatively simple expressions are derived for the probability of multiple hops in the exponential hopping limit. This limit is the one which is usually found in the diffusion of a metal atom on a metal surface. In this limit the barrier height (in units of $k_{\mathrm{B}} T$ ) is large compared with the bias created by the field and the energy loss of the particle as it traverses from one barrier to the next. The hopping distribution is obtained for constant and time varying fields in the adiabatic limit. Typically, the presence of an external field will increase the probability of long hops. The magnitude of the field needed to appreciably increase the probability of multiple hops is $10^{8}-10^{9} \mathrm{~V} \mathrm{~m}^{-1}$. (C) 1999 Elsevier Science B.V. All rights reserved.
\end{abstract}

Keywords: Atomistic dynamics; Computer simulations; Diffusion and migration; Energy dissipation; Equilibrium thermodynamics and statistical dynamics; Friction; Surface diffusion

\section{Introduction}

The dynamics of diffusion of a particle on a periodic potential is of interest for a variety of physical problems, such as surface diffusion of atoms and molecules [1,2], the current-voltage characteristics of superconducting devices [3], or the rotation of molecules in solids [4]. The 'standard' model studied by many authors is that of a particle with mass $m$ moving on a one-dimensional periodic potential $w(q)$ with 'lattice length' $l_{0}$, with barrier height $V^{\ddagger}$, whose equation of motion is the Langevin equation:

$m \ddot{q}+\frac{\mathrm{d} w(q)}{\mathrm{d} q}+m \gamma \dot{q}=\xi(t)$.

\footnotetext{
* Corresponding author. Fax: +972 89344123.

E-mail address: cfpollak@weizmann.weizmann.ac.il (E. Pollak)
}

The particle is subjected to a frictional force characterized by the friction coefficient $\gamma$ and a Gaussian delta-correlated random force $\xi(t)$ with zero mean such that at temperature $T$, $\langle\xi(t) \xi(\tau)\rangle=2 \gamma m k_{\mathrm{B}} T \delta(t-\tau)$.

If the barrier height $V^{\ddagger} \gg k_{\mathrm{B}} T$ then the diffusion is activated, and the particle must receive energy from the surrounding bath in order to surmount the barrier and escape from its present metastable state. The dynamics of activated diffusion has been studied extensively during the past decade [519,44]. The phenomenon of stochastic resonance [20] for periodic potentials is also of interest [2125]. Quantities of physical interest are the escape rate $\Gamma$ from a given well, the diffusion coefficient $D$ and the hopping probability distribution $p_{n}$. Upon escape, the particle may be retrapped in an adjacent well or it may be retrapped only after traversing over $n$ adjacent wells. The hopping 
distribution $\left(p_{n}\right)$ is defined as the probability for the particle to be retrapped in a well which is $n$ lattice lengths distant from it.

Mel'nikov [6] has provided analytic solutions for the escape rate, the diffusion coefficient and the hopping distribution as a function of the magnitude of the friction coefficient. For a given potential, the dynamics is characterized by the energy loss parameter $\Delta$, which is defined as the average energy lost by the particle as it traverses from one barrier to an adjacent one. When $\Delta \ll k_{\mathrm{B}} T$ one is in the underdamped regime, the characteristic jump length is long, of the order of $1 / \Delta$, the escape rate is proportional to $\Delta$ and the diffusion coefficient diverges as $1 / \Delta$. When the friction is 'low' but the energy loss is high such that $V^{\ddagger}>\Delta>k_{\mathrm{B}} T$, the total escape rate is given by the transition state theory limit $\Gamma=\left(\omega_{0} / \pi\right)$ $\exp \left(-V^{\ddagger} / k_{\mathrm{B}} T\right) \quad$ (where $\omega_{0}$ is the frequency of the adsorbed particle) and the hopping distribution is exponential in the energy loss $p_{n} \sim \exp \left[-(n-1) \Delta / 4 k_{\mathrm{B}} T\right]$. We shall refer to this situation as the exponential hopping regime. When the friction is high - the strong friction limit only single hops are allowed, the rate follows Kramers' law [26], it is inversely proportional to the friction coefficient and the diffusion coefficient is proportional to the product of the rate and the lattice length squared.

The hopping distribution has lately been a topic of special interest since it can be observed during the diffusion of metal atoms on metal surfaces. Various experimental groups have reported significant probabilities for multiple hops [1,2]. Their results have been interpreted in terms of Mel'nikov's theory $[14,15,19]$ and with the aid of molecular dynamics computations [27]. Typically, the friction felt by a diffusing atom is weak, although the energy loss is of the order of the thermal energy or higher. One is therefore in the exponential hopping regime, and one observes double or triple jumps; longer jumps are very improbable.

The topic of this paper is the control of the hopping distribution in the exponential hopping regime, by the use of an external field. If the force is very strong such that the field strength $F$ multiplied by the lattice length $l_{0}$ is greater than the barrier height for escape, the particle will no longer be trapped in a well and the process is no longer an activated one. We will consider only weak fields, in the sense that $F l_{0} \ll V^{\ddagger}$ and the particle is moving on a tilted potential. Mel'nikov provided an analytic solution for the diffusion dynamics in this case, but he did not study in any detail how the force affects the hopping distribution. In the presence of a constant force, the symmetry is broken, the particle will move 'downhill' and one will observe a current. At the same time though, the hopping distribution is modified. The first new result presented in this paper is that a constant force will also increase the probability for multiple hops. A simple expression for the modified multiple hopping probability in the physically relevant, exponential hopping regime is derived in Section 2.

If one applies an a.c. field, the Langevin equation is modified by the addition of a time-dependent periodic force $F f(t)$ where $F$ is the field strength and $f(t)$ oscillates with period $\tau$. Without loss of generality we assume that $|f(t)| \leq 1$. We will also assume that the time average of the force over one period is zero. If in addition, the time dependence is symmetric in the sense that there exists a time $t_{0}$ such that $f\left(t_{0}+t\right)=-f\left(t_{0}-t\right)$ for all $t$ (all odd moments of such a force are zero) then the particle will have equal probability of diffusing to the right or to the left. One remains with a purely diffusional process. However, the hopping distribution is modified by the symmetric field. We will show in Section 3, that the presence of the external symmetric field increases the probability of multiple hops and that accordingly the diffusion coefficient is increased.

Guided by recent work on the control of diffusion in the strong friction limit [28-33] we will also consider the case of a field that is periodic, but asymmetric in time. In the strong friction limit, it has been shown that such a field can induce a current; the symmetry of the process is broken through the asymmetry of the field. As demonstrated in Section 3, the same holds true in the exponential hopping regime. The asymmetric field induces a current but at the same time it modifies the hopping distribution. Finally we shall discuss 
the conditions under which such control of diffusion may be realized in atom diffusion on surfaces.

\section{Diffusion in the presence of a constant force}

As already mentioned in Section 1, in this paper we will consider the physically relevant exponential hopping distribution limit. In this limit, the reduced barrier height $v^{\ddagger} \equiv V^{\ddagger} / k_{\mathrm{B}} T \gg 1$ is the largest parameter of the problem. The magnitude of the friction coefficient is small $\left(\gamma / \omega_{0} \ll 1\right)$, but the reduced energy loss parameter $\delta \equiv \Delta / k_{\mathrm{B}} T$ is of the order of unity or larger. A positive constant force results from the addition of a linear potential term $-q F$ to the Hamiltonian of the system. The constant force tilts the potential. The (reduced) energy difference between left and right adjacent barriers will be denoted as $u$ and $u$ can be either positive or negative. We will assume weak fields, in the sense that $|u| \ll v^{\ddagger}$.

The particle may escape to the left with a rate $\Gamma_{1}$ or to the right with the rate $\Gamma_{\mathrm{r}}$. If the constant force is positive, the energy difference between adjacent barriers $u$ is taken to be positive and the escape rate to the right is higher than the escape rate to the left. One will have a net current of particles moving to the right. The rates are given by the TST expressions, which in the exponential hopping regime and under the weak field condition are well approximated as:

$\Gamma_{1}=\frac{\omega_{0}}{2 \pi} \exp \left[-\left(v^{\ddagger}+\frac{u}{2}\right)\right]$.

$\Gamma_{\mathrm{r}}=\frac{\omega_{0}}{2 \pi} \exp \left[-\left(v^{\ddagger}-\frac{u}{2}\right)\right]$.

The diffusion coefficient is proportional to the product of the rate and the mean squared jump length of an escaping particle:

$D=\frac{1}{2} \Gamma\left\langle l^{2}\right\rangle$,

where $\Gamma$ is the total rate out of the well $\left(\Gamma=\Gamma_{1}+\Gamma_{\mathrm{r}}\right)$ and the mean squared jump length is defined as:

$$
\left\langle l^{2}\right\rangle=l_{0}^{2} \sum_{j=1}^{\infty} j^{2} p_{j},
$$

where $p_{j}$ is the hopping distribution defined above.

Since the diffusion coefficient is proportional to the escape rate, the lowering of the barrier on the downhill side will cause an exponential increase of the diffusion coefficient. In contrast to a free particle, where the addition of a constant force does not change the diffusion coefficient, the presence of a nonlinear potential causes the external force to enhance the diffusion coefficient. This effect is the same as in the strong friction limit $[34,35]$. In the presence of strong time varying fields $\left(u>v^{\ddagger}\right)$, the nonlinear potential can cause the particle to diffuse faster than the free particle [30,31]. This is not the case in the weak field limit considered in this paper.

The external force also changes the hopping distribution and thus causes a further increase of the diffusion coefficient. In the exponential hopping regime, the energy distribution of escaping particles is thermal. As a particle moves from one barrier to the next, the effect of the environment is to cause a fluctuation in the energy. As shown by Mel'nikov [6], in the weak field limit, the conditional probability that the particle changes its (reduced) energy $\left(\epsilon \equiv E / k_{\mathrm{B}} T\right)$ from $\epsilon$ to $\epsilon^{\prime}$ as it traverses from one barrier to the adjacent one is a Gaussian:

$P\left(\epsilon^{\prime} \mid \epsilon\right)=\frac{1}{\sqrt{4 \pi \delta}} \exp \left[-\frac{\left(\epsilon^{\prime}-\epsilon-u+\delta\right)^{2}}{4 \delta}\right]$.

The fraction of particles that start at a barrier with positive (negative) momentum and reach the adjacent barrier whose relative height is $u(-u)$ is given by (the barrier energy is zero):

$$
\begin{aligned}
F_{2,1}(\delta, u) & =\int_{0}^{\infty} \mathrm{d} \epsilon \int_{0}^{\infty} \mathrm{d} \epsilon^{\prime} P\left(\epsilon \mid \epsilon^{\prime}\right) \exp \left(-\epsilon^{\prime}\right) \\
& =\frac{1}{2}\left[\operatorname{erfc}\left(\frac{\delta-u}{2 \sqrt{\delta}}\right)+\mathrm{e}^{u} \operatorname{erfc}\left(\frac{\delta+u}{2 \sqrt{\delta}}\right)\right] .
\end{aligned}
$$

In the absence of an external force $(u=0)$, this fraction is independent of the direction of the jump. In the presence of an external force, $u \neq 0$, 
the fraction to the right $F_{2,1}(\delta, u)$ will differ from the fraction to the left $F_{2,1}(\delta,-u)$.

The probability for single jumps is by definition:

$p_{1}(\delta, u)=\frac{\Gamma-\Gamma_{\mathrm{r}} F_{2,1}(\delta, u)-\Gamma_{1} F_{2,1}(\delta,-u)}{\Gamma}$.

and the multiple hopping probability is:

$$
\begin{aligned}
p_{j \geq 2}(\delta, u) & =1-p_{1}(\delta, u) \\
& =\frac{\Gamma_{\mathrm{r}} F_{2,1}(\delta, u)+\Gamma_{1} F_{2,1}(\delta,-u)}{\Gamma} .
\end{aligned}
$$

The fraction of particles that start at the barrier top with positive (negative) momentum and are immediately trapped in the well to the right (left) is:

$p_{1, \mathrm{r}(1)}=1-F_{2,1}(\delta, \pm u)$.

The enhancement of the multiple hopping probability as a result of the bias is given by the ratio of the multiple hopping probability in the presence of the field to the probability in the absence of the field:

$r_{j \geq 2}=\frac{p_{j \geq 2}(\delta, u)}{p_{j \geq 2}(\delta, 0)}$.

It is straightforward to obtain the fraction of particles that start at a barrier with positive (negative) momentum and jump to a barrier which is distant by at least two lattice lengths. Following the derivation of Eq. (7), one finds that:

$$
\begin{aligned}
F_{3,1}(\delta, u)= & \int_{0}^{\infty} \mathrm{d} \epsilon \int_{0}^{\infty} \mathrm{d} \epsilon^{\prime} \int_{0}^{\infty} \mathrm{d} \epsilon^{\prime \prime} P\left(\epsilon \mid \epsilon^{\prime}\right) P\left(\epsilon^{\prime} \mid \epsilon^{\prime \prime}\right) \mathrm{e}^{-\epsilon \prime \prime} \\
= & \frac{\mathrm{e}^{-\delta / 2} \mathrm{e}^{u}}{4} \int_{0}^{\infty} \mathrm{d} x\left[\operatorname{erf}\left(\frac{x-u}{\sqrt{2 \delta}}\right)\right. \\
& \left.+\operatorname{erf}\left(\frac{x+u}{\sqrt{2 \delta}}\right)\right] \operatorname{erfc}\left(\frac{-x}{\sqrt{2 \delta}}\right) \mathrm{e}^{-x}
\end{aligned}
$$

The probability for double jumps is then:

$$
\begin{aligned}
& p_{2}(\delta, u) \\
& =\frac{\Gamma_{\mathrm{r}}\left[F_{2,1}(\delta, u)-F_{3,1}(\delta, u)\right]+\Gamma_{1}\left(F_{2,1}(\delta,-u)-F_{3,1}(\delta,-u)\right]}{\Gamma} .
\end{aligned}
$$

and the probability for double jumps to the right (left) is:

$p_{2, \mathrm{r}(1)}=F_{2,1}(\delta, \pm u)-F_{3,1}(\delta, \pm u)$.

The enhancement of the double hopping probability is by definition:

$r_{2}=\frac{p_{2}(\delta, u)}{p_{2}(\delta, 0)}$.

In this manner, one can continue and derive the complete hopping distribution.

In Table 1 we compare theory with numerical simulation for a particle with unit mass and the potential $w(q)=\cos q$ at the temperature of $\beta=10$ such that the reduced barrier height is $v^{\ddagger}=20$. The reduced friction coefficient is fixed at $\gamma / \omega_{0}=0.1$ so that the reduced energy loss parameter is $\delta=8$. These parameters mimic roughly the diffusion of $\mathrm{Pt}$ on $\operatorname{Pt}(110)$, where the observed fraction of double jumps in the absence of an external bias is of the order of 0.1 or less. The numerically exact computations were performed using the reactive flux method [13,35-38] and the 4th-order Hershkovitz integrator [39]. Results are given for the probability for double jumps (Eq. (14)) as a function of the bias parameter $u$. As can be seen, the agreement is quantitative.

The enhancement of the multiple hopping probability (Eq. (11)) as a function of the bias $u$ is plotted in Fig. 1. We note that the multiple hopping probability may be doubled already when $u \sim 2$. The enhancement of the diffusion coefficient $(D(u) / D(0))$ is plotted in Fig. 2 (thick solid line) as a function of the bias $u$. As already mentioned, the enhancement comes from two sources: one is the enhancement of the escape rate, the other is the increase in the

Table 1

Hopping probabilities in the presence of a constant force

\begin{tabular}{lllll}
\hline$u$ & $p_{1, \mathrm{r}}^{\mathrm{a}}$ & $p_{1,1}^{\mathrm{a}}$ & $p_{2, \mathrm{r}}^{\mathrm{a}}$ & $p_{2,1}^{\mathrm{a}}$ \\
\hline$\pi / 5$ & $.938(.94)$ & $.967(.97)$ & $.0557(.055)$ & $.0312(.03)$ \\
$\pi / 2$ & $.906(.91)$ & $.980(.98)$ & $.0799(.081)$ & $.0190(.018)$ \\
$0.6 \pi$ & $.892(.89)$ & $.9837(.98)$ & $.0889(.091)$ & $.0159(.015)$ \\
$\pi$ & $.826(.83)$ & $.9925(.99)$ & $.127(.133)$ & $.0074(.007)$ \\
$1.2 \pi$ & $.784(.79)$ & $.9950(.98)$ & $.144(.153)$ & $.0049(.004)$ \\
\hline
\end{tabular}

${ }^{a}$ The first number in the column is the analytical result based on Eq. (14). The number in parentheses is the result from numerical simulation. 


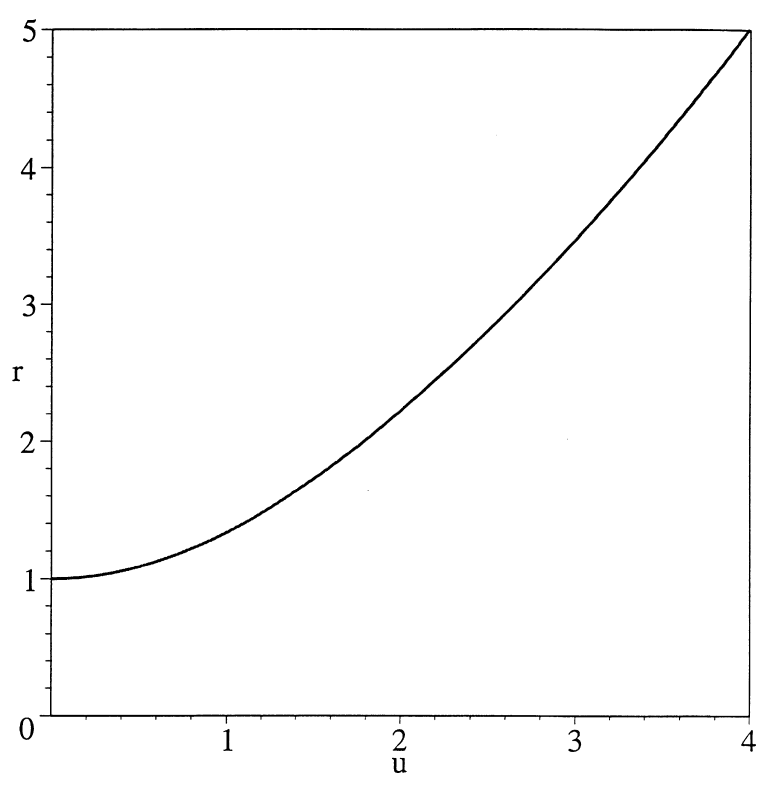

Fig. 1. Enhancement of the multiple hopping probability in the presence of a constant force. The ratio $r_{2}$ defined in Eq. (11) is plotted versus $u$ the reduced energy difference between successive barriers. The energy loss parameter is $\delta=8$.

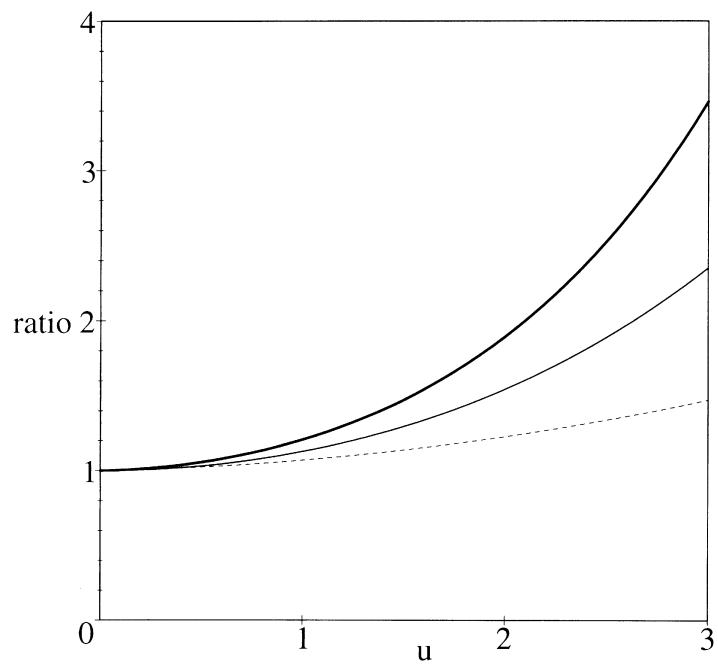

Fig. 2. Enhancement of the diffusion coefficient in the presence of a constant force. The ratio $r$ of the diffusion coefficient in the presence and the absence of a field is plotted (thick solid line) versus $u$ - the reduced energy difference between successive barriers of the potential. Also plotted in the figure is the enhancement of the rate (thin solid line) and the mean squared jump length (dashed line). probability for multiple hops. To get a feeling for the relative contribution of the two effects, we also plot in the figure the enhancement of the rate $(\Gamma(u) / \Gamma(0)$, thin solid line) and the enhancement of the mean squared jump length $\left(\left\langle l^{2}\right\rangle(u) /\left\langle l^{2}\right\rangle(0)\right.$, dashed line). In this plot, the diffusion coefficient and the mean squared jump length were obtained by allowing only up to triple jumps, since the probability for longer jumps is negligible within the present parameter range.

\section{Time-dependent fields}

\subsection{The symmetric time-dependent force case}

The next case to be considered is that of a symmetric periodic force. It is straightforward to estimate the hopping distribution in the adiabatic limit. In this limit, the periodic force changes slowly when compared with the time it takes the particle to escape from the well (the inverse TST rate). One may then define time-dependent rates, obtained for the biased periodic potential with the constant force $F f(t)$. The full rates are obtained by averaging the time-dependent rates over one period of the force. If the force is symmetric, the time average of the hopping rates to the left and to the right will be identical. The absolute value of the escape rate as well as the multiple hopping probabilities will be increased relative to the multiple hopping ratio in the absence of the force, since the downhill part of the force will enhance the multiple hops as before, while the uphill part is less probable because of the increased barrier height.

The enhancement of the time averaged multiple hopping probability is defined as: (cf. Eq. (11)):

$\left\langle r_{j \geq 2}\right\rangle=\frac{1}{\tau} \int_{0}^{\tau} \mathrm{d} t \frac{p_{j \geq 2}(\delta, u(t))}{p_{j \geq 2}(\delta, 0)}$,

where $\tau$ is the period of the symmetric field and $u(t)$ is the instantaneous (reduced) energy difference between adjacent barriers. In the weak field limit, $u(t) \simeq u f(t)$ where $u>0$ is the maximal reduced energy difference between two adjacent barriers. 
In Fig. 3 we show the adiabatic results obtained for $f(t)=\cos (\Omega t)$, keeping the same parameters as in Figs. 1 and 2. Comparing Figs. 1 and 3, one notes that the enhancement of multiple hops in the presence of the a.c. field is similar to the d.c. field case. As might be expected, the a.c. field does reduce somewhat the effect, as a result of the averaging.

If the frequency of the field $\Omega$ is fast compared with the characteristic frequency of the diffusing particle $\omega_{0}$ then the field averages out to zero and there is no effect. Between this mean field limit and the adiabatic limit there can be variations of the field frequency over many orders of magnitude, as the typical reduced barrier height $v$ is assumed to be large. To get a feeling for the effect of an external force in this intermediate frequency range it is necessary to carry out numerical simulations. However, even this is not trivial. If the reduced barrier height is large, it will take a long time for a trajectory initiated at the bottom of a well to escape. In the absence of a time-dependent field one overcomes this problem by using the reactive

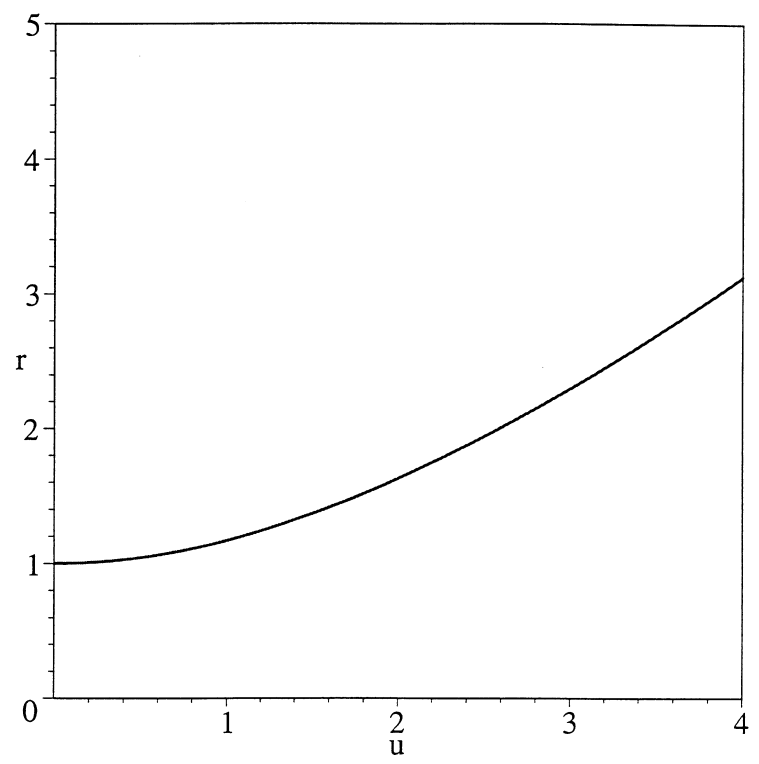

Fig. 3. Enhancement of the multiple hopping probability in the presence of a slowly varying symmetric periodic force $-f(t)=$ $\cos (\Omega t)$. The enhancement, computed in the adiabatic limit (see text) is plotted versus $u$ - the (reduced) maximal height difference between successive barriers. flux method which allows one to initiate trajectories at the barrier. However, the reactive flux method is not well defined for time-dependent Hamiltonians, as there is no stationary equilibrium probability. A more extensive study of the numerically exact diffusion dynamics in the presence of a time-dependent field remains at present a challenge for future work.

\subsection{The asymmetric time-dependent force case}

In the adiabatic limit, one can just as well treat the case of an asymmetric force. For each instant $t$ one has an escape rate $\Gamma_{ \pm}(t)$ to the right or to the left given by the TST expression:

$\Gamma_{\mathrm{r}(1)}(t)=\frac{\omega_{0}}{2 \pi} \exp \left[-\left(v^{\ddagger} \mp \frac{u(t)}{2}\right)\right]$,

where $u(t)$ is the instantaneous adjacent barrier height difference. The net rate in each direction is then the time averaged rate:

$\left\langle\Gamma_{\mathrm{r}(1)}\right\rangle=\frac{1}{\tau} \int_{0}^{\tau} \mathrm{d} t \Gamma_{\mathrm{r}(1)}(t)$,

where $\tau$ is the period of the asymmetric force.

Consider for simplicity the time-dependent force:

$f(t)=f_{1}\left[\frac{\tau}{t_{1}} \theta\left(t_{1}-t\right)-\frac{\tau}{\tau-t_{1}} \theta\left(t-t_{1}\right)\right]$,

defined over the period $0 \leq t \leq \tau$, such that $t_{1}<\tau / 2$. The absolute magnitude of the force will be greater in the short interval $t_{1}$ than in the long interval $\tau-t_{1}$. The averaged rate depends exponentially on the magnitude of the slope, but only linearly on the time the force is exerted. Therefore, the main contribution to the rate will come from the strong force. This will bias the rate in favor of the high negative slope direction and a net current will be created. The magnitude of the current is proportional to the difference between the uphill and downhill rates. The efficiency of the asymmetric field, denoted by $e$ is defined as:

$e=\frac{\left|\left\langle\Gamma_{\mathrm{r}}\right\rangle-\left\langle\Gamma_{1}\right\rangle\right|}{\left\langle\Gamma_{\mathrm{r}}\right\rangle+\left\langle\Gamma_{1}\right\rangle}$. 
In practice it is easier to prepare a continuous asymmetric time-dependent field. We therefore used the following form, plotted in Fig. 4:

$f(t)=\frac{2}{3}\left[\cos (\Omega t)+\frac{1}{2} \cos (2 \Omega t)\right]$.

The efficiency of the field is plotted in Fig. 5 as a function of the field strength, as represented by the barrier energy difference $u$, which is the maximal height difference between adjacent barriers. As is evident, the typical efficiency is of the order of $10^{-2}-10^{-1}$. The enhancement of the multiple hopping probability is plotted in Fig. 6.

\section{Discussion}

The central question which must be answered is whether the proposed control mechanism can actually be implemented experimentally. In this paper we consider specifically the case of activated surface diffusion of adatoms on surfaces. As shown by Kandel and Kaxiras [40], because the electronic structures of the barriers and wells of the surface are different, the electronic cloud at the surface induces an effective small charge of the order of $0.1 e$ on the adatom. An electric field can then

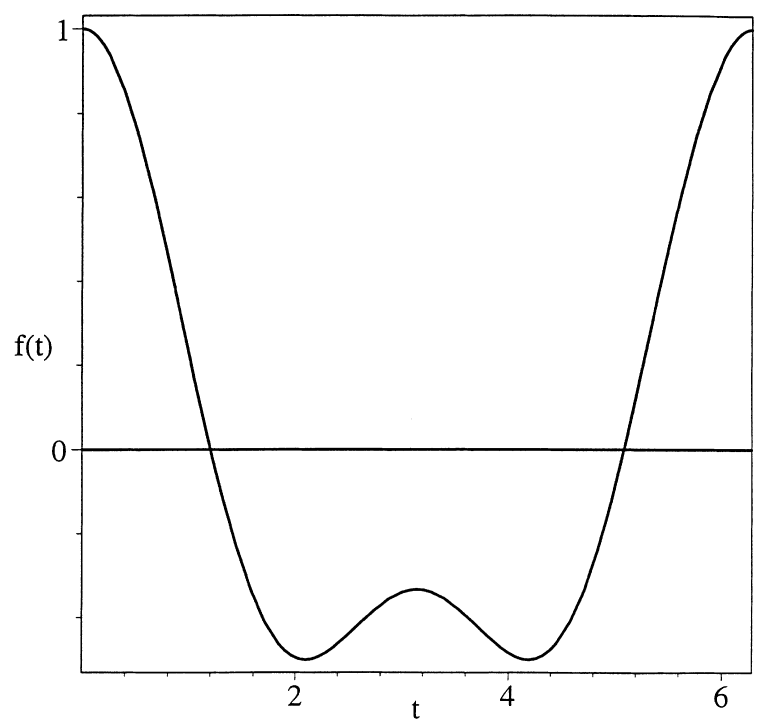

Fig. 4. An asymmetric periodic force. The asymmetric periodic force given in Eq. (21) is plotted versus the time $t$, measured in units of $\Omega^{-1}$.

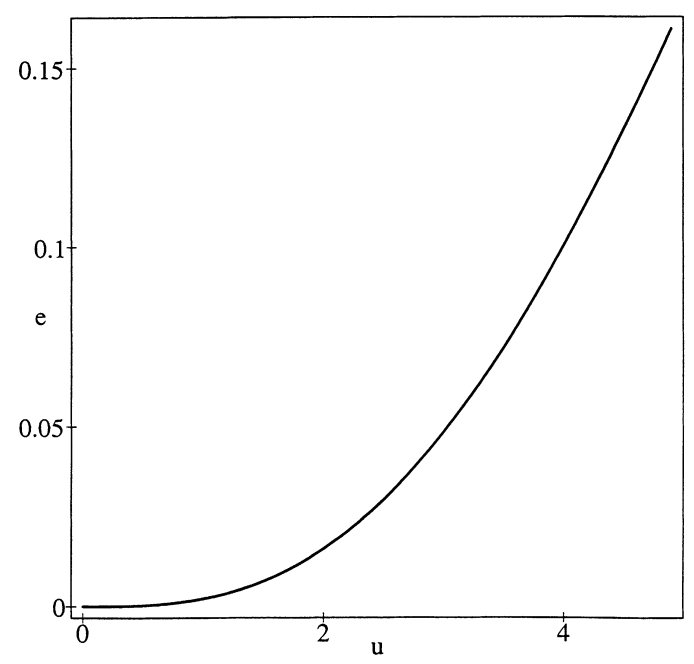

Fig. 5. The efficiency of the asymmetric periodic force in inducing a net current. The efficiency defined in Eq. (20) is plotted versus the reduced bias parameter $u$ defined as the maximal energy difference between successive barriers. The results are in the adiabatic limit of a slowly varying asymmetric field.

control the motion of the atom. A back of the envelope computation for the field strength needed to cause a bias of $u=1$ (in reduced units) is $10^{8}-10^{9} \mathrm{~V} \mathrm{~m}^{-1}$. This is a very strong field when compared with characteristic breakdown in insula-

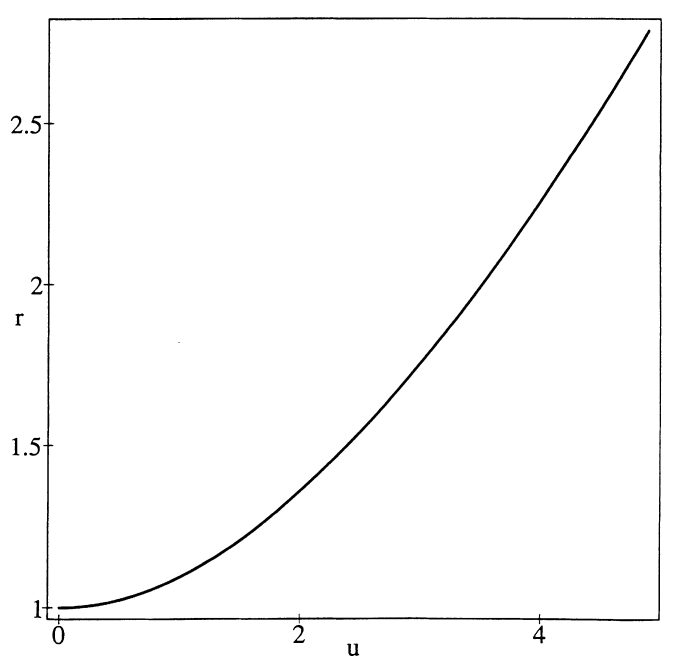

Fig. 6. The enhancement of the multiple hopping probability (Eq. (16)) in the presence of an adiabatically varying asymmetric field. The enhancement is plotted versus the reduced bias parameter $u$. All other details are as in Fig. 5. 
tors, which occurs at $10^{5}-10^{6} \mathrm{~V} \mathrm{~m}^{-1}$. It is, however, typical of field strengths in field ion microscopy $[41,42]$. Such fields are also obtainable when using an STM tip as demonstrated recently by Carpinelli and Swartzentruber [43]. We also know that STM tips may be used to pull adatoms from a surface and then to redeposit them elsewhere. The more difficult question is how does one measure the diffusion dynamics in the presence of the tip. A two-tip experiment seems to be impossible at this time.

The study presented here poses some interesting theoretical challenges. Can one come up with a robust numerical algorithm for computing the hopping probabilities in the presence of a timedependent field? Alternatively, is it possible to generalize the reactive flux method so that it is applicable to the time-dependent field case? In this paper we studied a one-dimensional model. A diffusing particle moves in three-dimensional space. How the added dimensions would affect the field control is not clear. The present study has focused on the classical diffusion dynamics. We know that quantum effects may be significant when considering the diffusion of a light particle such as a hydrogen atom $[9,12]$. The quantum hopping distribution is different from the classical: quantum tunneling and reflection tend to suppress multiple hops. Is an external field more or less effective in the quantum regime? These questions remain open and will hopefully be answered in future work on this problem.

\section{Acknowledgements}

We thank the anonymous referees for their helpful comments. This work was supported by grants of the Minerva Foundation, Munich/ Germany (E.P.), the German-Israeli Foundation for Scientific Research and Development (E.P. and P.H.) and a Meitner-Humboldt Research Award (E.P.).

\section{References}

[1] D.C. Senft, G. Ehrlich, Phys. Rev. Lett. 74 (1995) 294.

[2] T.R. Linderoth, S. Horch, E. Lægsgaard, I. Stensgaard, F. Besenbacher, Phys. Rev. Lett. 78 (1997) 4978.
[3] E. Turlo, D. Esteve, C. Urbina, J.M. Martinis, M.H. Devoret, S. Linkwitz, H. Grabert, Phys. Rev. Lett. 62 (1989) 1788.

[4] Y. Georgievskii, A. Burshtein, J. Chem. Phys. 100 (1994) 7319.

[5] Z. Zhang, K. Haug, H. Metiu, J. Chem. Phys. 93 (1990) 3614.

[6] V.I. Mel'nikov, Phys. Rep. 209 (1991) 1.

[7] K. Haug, H. Metiu, J. Chem. Phys. 94 (1991) 3251.

[8] K.D. Dobbs, D.J. Doren, J. Chem. Phys. 97 (1992) 3722.

[9] E. Pollak, J. Bader, B.J. Berne, P. Talkner, Phys. Rev. Lett. 70 (1993) 3299.

[10] R. Ferrando, R. Spadacini, G.E. Tommei, Phys. Rev. E 48 (1993) 2437.

[11] R. Ferrando, R. Spadacini, G.E. Tommei, G. Caratti, Surf. Sci. 311 (1994) 411.

[12] Y. Georgievskii, E. Pollak, Phys. Rev. E 49 (1994) 5098.

[13] J.S. Bader, B.J. Berne, E. Pollak, J. Chem. Phys. 102 (1995) 4037.

[14] Y. Georgievskii, M.A. Kozhushner, E. Pollak, J. Chem. Phys. 102 (1995) 6908.

[15] Y. Georgievskii, E. Pollak, Surf. Sci. 355 (1996) L366.

[16] G. Caratti, R. Ferrando, R. Spadacini, G.E. Tommei, Phys. Rev. E 54 (1996) 4708.

[17] L.Y. Chen, M.R. Baldan, S.C. Ying, Phys. Rev. B 54 (1996) 8856.

[18] S.Yu. Krylov, A.V. Prosyanov, J.J.M. Beenakker, J. Chem. Phys. 107 (1997) 6970.

[19] E. Hershkovitz, P. Talkner, E. Pollak, Y. Georgievskii, Surf. Sci. 421 (1999) 73.

[20] L. Gammaitoni, P. Hänggi, P. Jung, F. Marchesoni, Rev. Mod. Phys. 70 (1998) 223.

[21] G. Hu, Phys. Lett. A 174 (1993) 247.

[22] M. Gitterman, I.B. Khalfin, B.Ya. Shapiro, Phys. Lett. A 184 (1994) 340.

[23] J.M. Casado, J.J. Mejias, M. Morillo, Phys. Lett. A 197 (1995) 365.

[24] F. Marchesoni, Phys. Lett. A 231 (1997) 61.

[25] Y.W. Kim, W. Sung, Phys. Rev. E 57 (1998) R6237.

[26] H.A. Kramers, Physica 7 (1940) 284.

[27] J. Jacobsen, K.W. Jacobsen, J.P. Sethna, Phys. Rev. Lett. 79 (1997) 2843.

[28] P. Jung, P. Hänggi, Ber. Bunsenges. Phys. Chem. 95 (1991) 311.

[29] I. Claes, C. Van den Broeck, J. Stat. Phys. 70 (1993) 1215.

[30] Hu. Gang, A. Daffentshofer, H. Haken, Phys. Rev. Lett. 76 (1996) 4874.

[31] M. Schreier, P. Reimann, P. Hänggi, E. Pollak, Europhys. Lett. 44 (1998) 416.

[32] I. Goychuk, M. Grifoni, P. Hänggi, Phys. Rev. Lett. 81 (1998) 649.

[33] I. Goychuk, P. Hänggi, Europhys. Lett. 43 (1998) 503.

[34] R.L. Stratonovich, Topics in the Theory of Random Noise Vol. II, Gordon and Breach, New York, 1967.

[35] P. Hänggi, P. Talkner, M. Borkovec, Rev. Mod. Phys. 62 (1990) 251.

[36] A.F. Voter, J.D. Doll, J. Chem. Phys. 82 (1985) 80. 
[37] A.F. Voter, J.D. Doll, J.M. Cohen, J. Chem. Phys. 90 (1989) 2045.

[38] B.J. Berne, in: P. Hänggi, G.R. Fleming (Eds.), Activated Barrier Crossing, World Scientific, New York, 1993.

[39] E. Hershkovitz, J. Chem. Phys. 108 (1998) 9253.

[40] D. Kandel, E. Kaxiras, Phys. Rev. Lett. 76 (1996) 1114.
[41] R. Gomer, Rep. Prog. Phys. 53 (1990) 917.

[42] T. Tsong, G. Kellogg, Phys. Rev. B 12 (1975) 1343.

[43] J.M. Carpinelli, B.S. Swartzentruber, Phys. Rev. B 58 (1998) 13423.

[44] G. Caratti, R. Ferrando, R. Spadacini, G.E. Tommei, Chem. Phys. 235 (1998) 157. 